\title{
The Density of States of the Two Dimensional Electron Gas in InAs/AISb Quantum Well
}

\author{
Baymatov Paziljon Jamoldinovich*, Bahrom Toshmirza O'g'li Abdulazizov
}

Department of Physics, Namangan State University, Namangan, Republic of Uzbekistan

Emailaddress:

baymatov1958@mail.ru (B. P. Jamoldinovich), bt_abdulazizov@mail.ru (B. T. O. Abdulazizov)

${ }^{*}$ Corresponding author

To cite this article:

Baymatov Paziljon Jamoldinovich, Bahrom Toshmirza O'g'li Abdulazizov. The Density of States of the Two Dimensional Electron Gas in InAs/AlSb Quantum Well. American Journal of Physics and Applications. Vol. 6, No. 1, 2018, pp. 18-21. doi: 10.11648/j.ajpa.20180601.14

Received: November 24, 2017; Accepted: December 6, 2017; Published: January 11, 2018

\begin{abstract}
It is considered the possibility of determining the density of states of the two-dimensional electron gas as a function of total concentration $n_{s}$ on the basis of some experimental data. For this purpose it is used the known value of the effective mass at the Fermi level from the cyclotron resonance measurements and the number of quantized levels located below the Fermi level from the measurements of the Shubnikov-de Haas. The obtained density of states is compared with model calculation that takes into account the non-parabolic conduction band. The experimental data and model calculations correspond to the hetero structured quantum well InAs/AlSb with width of $15 \mathrm{~nm}$.
\end{abstract}

Keywords: Quantum Well, Kane Model, Subband Dispersion, InAs/AlSb Hetero Structure, Two-Dimensional Electron Gas, Density of States, Effective Mass

\section{Introduction}

In recent years, much attention is paid to the study of the properties of two-dimensional electron gas in a semiconductor hetero structure quantum well $(\mathrm{QW})$. These structures appear to be quite promising for solid-state electronics. Spatial quantized energy levels of carriers in the QW leads to a significant change in their dispersion form and therefore the density of states (DOS) of gas as a function of energy. In the two-dimensional gas of free electron DOS is changing abruptly by energy [1].

Hetero structures on the basis of InAs semiconductors have been intensively studied recently [2-4]. Those semiconductors are characterized by a strong nonparabolicity of their conduction bands. However, only undoped hetero structures with two-dimensional (2D) electron concentrations up to $10^{12} \mathrm{~cm}^{-2}$ were examined in these works, i.e. electron concentration there was small. Nevertheless, the occupation by the electrons of more than one subbands in the selectivelydoped InAs/AlSb QW is experimentally possible [5].

Currently, there are several experimental methods for DOS studying of 2D electrons in the QW. Methods of DOS studying of $2 \mathrm{D}$ electron gas in a transverse magnetic field are described in [6]. In work [7] it was studied the possibility of finding the electron DOS in the QW and the ensembles of quantum dots in the hetero structures of the current-voltage characteristics.

In this paper is considered the definition of the DOS at the Fermi level in the 2D electron gas as a function of total concentration $n_{s}$. This requires the value of the transport effective mass at the Fermi level $m_{c}\left(E_{F}\right)$ and the number of quantized levels $M$ lying below the Fermi level. The effective mass $m_{c}\left(E_{F}\right)$ can be determined from measurements of cyclotron resonance (CR), and the value $M$ -from the measurements of the Shubnikov-de-Haas (ShdH) [5]. The total concentration of $2 \mathrm{D}$ electron gas $n_{s}$ can be determined from Hall measurements.

\section{Determination of the DOS at the Fermi Level}

Let's consider the degenerate ideal Fermi gas with the spectrum $\varepsilon_{\mathbf{k}}$. The broadening of levels due to irregularities will not be considered. The dependence of the DOS on the energy can be written as 


$$
\rho(E)=2 \sum_{\mathbf{k}} \delta\left(\varepsilon_{\mathbf{k}}-E\right)
$$

For an electron gas in the QW summation on $k_{z}$ will be replaced by a summation over the quantum levels and integration on $k_{x}$ and $k_{y}$. Using properties of $\delta$-function for DOS of 2D electron gas from (1) can be obtained following expression

$$
\begin{array}{r}
\rho_{s}(E)=\frac{1}{2 \pi} \sum_{i=1} \frac{d k^{2}}{d E} \theta\left(E-E_{i}(0)\right) \quad \text { (2) In case } E=E_{F}, \text { from (2) and } \\
\rho_{s}\left(E_{F}\right)=\rho_{0} \sum_{i=1}^{\infty} \frac{m_{c}\left(E_{F}\right)}{m_{0}} \theta\left(E_{F}-E_{i}(0)\right), \rho_{0}=\frac{m_{0}}{\pi \hbar^{2}}=\frac{413 \cdot 10^{12}}{\mathrm{e} V \cdot \mathrm{sm}^{2}}
\end{array}
$$

Here, i-subband index, $\theta(x)$-step function, $E_{n}(0)$ quantized levels electron energy in the QW. The values $d k^{2} / d E$ and $E_{i}(0)$ can be determined by solving the quantum mechanical problem of a single electron in the QW with allowance for the non-parabolic of band structure of InAs/AlSb. On the other hand, the value $d k^{2} / d E$ is associated with transport mass according to the expression

$$
m_{c}(E)=\hbar^{2} k d k / d E
$$

In case $E=E_{F}$, from (2) and (3) follows
From investigations [5, 8-11] follows, that in the QW hetero structure based on narrow-gap semiconductors as the $\mathrm{InAs} / \mathrm{AlSb}$ or InAsSb/AlSb transport effective mass of an electron in the QW $m_{c}\left(E_{F}\right)$ strongly depends Fermi energy, however, on the Fermi level, it is weakly dependent on the number of subband $i$. Only under this condition, formula (4) can be written as

$$
\rho_{s}\left(E_{F}\right) \approx \rho_{0} \frac{m_{c}\left(E_{F}\right)}{m_{0}} M
$$

where $M=\sum_{i=1}^{\infty} \theta\left(E-E_{i}(0)\right)$ is the number of quantized levels lying below the Fermi level, $m_{0}$-the free electron mass.

Table 1. Concentration dependence of the effective mass [5]: InAs/AlSb.

\begin{tabular}{lllll}
\hline № & $\mathbf{n}_{\mathbf{s}}, \mathbf{1 0} \mathbf{c m}^{\mathbf{1 2}}$ & $\mathbf{M}$ & $\mathbf{m}_{\mathrm{c}} / \mathbf{m}_{\mathbf{0}}$ & $\boldsymbol{\rho}_{\mathbf{s}}, \mathbf{1 0} \mathbf{1 0}^{\mathbf{1 2}} \mathbf{c m}^{\mathbf{- 2}}$ \\
\hline 1 & $0.27(\mathrm{pc})$ & 1 & 0.030 & 12.39 \\
2 & $0.63(\mathrm{pc})$ & 1 & 0.034 & 14.04 \\
3 & $0.67(\mathrm{pc})$ & 1 & 0.035 & 14.46 \\
4 & $0.82(\mathrm{pc})$ & 1 & 0.036 & 14.87 \\
5 & $2.4(\mathrm{~h})$ & 2 & 0.043 & 35.52 \\
6 & $3.2(\mathrm{~h})$ & 2 & 0.043 & 35.52 \\
7 & $4.3(\mathrm{~h})$ & 2 & 0.046 & 38.00 \\
8 & $8.3(\mathrm{~h})$ & 3 & 0.057 & 70.62 \\
\hline
\end{tabular}

pc-from oscillations of submillimeter photoconductivity h-from the Hall effect measurements.

Table 1 shows the parameters of the electron gas in the undoped (1-4) and selectively doped (5-8) samples from the experiment [5]. The value $M$ can be determined by the presence of non-zero concentration of electrons in the subbands $n_{s}^{(i)}, \mathrm{i}=1,2,3 \ldots$, from measurements [5]. The last column of Table 1 shows the values of DOS calculated using the formula (5).

\section{Simplified Model of the Spectrum and the Calculation of the DOS}

Simulated structure is represented as a rectangular QW with width L (area A-InAs), concluded between the potential barrier with height $\mathrm{V}$ (area B-AlAs). The energy is measured from the bottom of the band of bulk InAs. In the single-band approximation the solution of three-dimensional Schrödinger equation leads to dispersion $E(k, i)$ [11]

$$
E_{A}=E_{0}\left(\pi \cdot i-2 \arcsin \frac{1}{\sqrt{1+\eta}}\right)^{2}
$$

Here, $E_{A}=E-\hbar^{2} k^{2} / 2 m_{A} \quad, \quad E_{B}=E-\hbar^{2} k^{2} / 2 m_{B}$, $E_{0}=\hbar^{2} / 2 m_{A} L^{2}, k^{2}=k_{x}^{2}+k_{y}^{2}, \eta=\left(m_{A} / m_{B}\right)\left(V-E_{B}\right) / E_{A}$, $m_{A, B}$-energy-dependent effective mass of the electrons in the material A or B. In this work the non-parabolicity of the conduction band is described with simplified Kane model

$$
m_{A}=m_{A}(0)\left(1+\frac{E}{E_{g A}}\right), m_{B}=m_{B}(0)\left(1+\frac{E-V}{E_{g B}}\right)
$$

Here, $E_{g A}$ and $E_{g B}$-the forbidden bands InAs and AlSb respectively. To further simplify rewrite the dispersion equation (6) in the form

$$
E\left(1+\frac{E}{E_{g A}}\right)=\frac{\hbar^{2} k^{2}}{2 m_{A}(0)}+\frac{\hbar^{2}}{2 m_{A}(0) L^{2}}\left(\pi \cdot i-2 \arcsin \frac{1}{\sqrt{1+\eta}}\right)^{2}
$$

In case $k=0$ from (8) can be obtained the equation for determining the quantized energy levels (the bottom of the $i$ th subband) $E_{i}=E(0, i)$

$$
E_{i}\left(1+\frac{E_{i}}{E_{g A}}\right)=\frac{\hbar^{2}}{2 m_{A}(0) L^{2}}\left(\pi \cdot i-2 \arcsin \frac{1}{\sqrt{1+\eta_{0}}}\right)^{2}
$$

where $\eta_{0}=\left(m_{A}\left(E_{i}\right) / m_{B}\left(E_{i}\right)\right)\left(V-E_{i}\right) / E_{i}$. Numerical tests have shown that when $k$ changes the second term on the right side (8), varies much more slowly than the first. If 
neglect this weak dependence and take its value at the point $k=0$, then from (9) can be obtained the following approximation

$$
E\left(1+\frac{E}{E_{g A}}\right) \approx \frac{\hbar^{2} k^{2}}{2 m_{A}(0)}+E_{i}\left(1+\frac{E_{i}}{E_{g A}}\right)
$$

The values $E_{i}$ is calculated from equations (9) for the given parameters of materials. For InAs/AlSb QW it is possible will check up graphically (see below), that the approximation (10) accurately describes the solution of the original equation (8) in a wide range of values of $L$.

Taking into account (10) for the transport mass (3) can be obtained the following formula (here in after assumed $E=E_{F}$ )

$$
m_{c}\left(E_{F}\right)=m_{c}(0)\left(1+2 \frac{E_{F}}{E_{g A}}\right)
$$

For the DOS at the Fermi level from (2) follows the formula

$$
\rho_{s}\left(E_{F}\right)=\rho_{0} \frac{m_{c}(0)}{m_{0}}\left(1+2 \frac{E_{F}}{E_{g A}}\right) \sum_{i=1}^{\infty} \theta\left(E_{F}-E_{i}(0)\right)
$$

It can be shown [11] that, for dispersion (10) the total 2D electron concentrations $n_{s}$ can be written as

$$
n_{s}=\rho_{0} \sum_{i=1}\left(E_{F}+\frac{E_{F}^{2}}{E_{g A}}-E_{i}-\frac{E_{i}^{2}}{E_{g A}}\right) \theta\left(E_{F}-E_{i}(0)\right)
$$

The system of equations (12) and (13) allows finding the DOS depending from the total concentration $\rho_{s}\left(n_{s}\right)$, and the system of equations (11) and (13) allows finding the depending of effective mass at the Fermi level from the concentration $m_{c}\left(n_{s}\right)$. In the limit $E_{g A} \rightarrow \infty$ from the relations (11), (12) and (13) follows a formula for the parabolic bands.

\section{Numerical Results and Discussion}

First, from the equation (9) it is necessary define the values of the quantized energy levels. In calculations used the following parameters of materials InAs and AlSb: value of band gap $\mathrm{E}_{\mathrm{g}}$, the effective mass $\mathrm{m}(0)$ and conduction band jump $V$. The values of these parameters are given in Table 2 .

Table 2. The parameters of InAs/AlSb QW.

\begin{tabular}{lll}
\hline & InAs(A) & AlSb(B) \\
\hline$E_{\mathrm{g}}, \mathrm{eV}$ & 0.42 & 2.37 \\
$m(0) / m_{0}$ & 0.023 & 0.11 \\
$\mathrm{~V}(\mathrm{z}), \mathrm{eV}$ & 0 & 1.35 \\
\hline
\end{tabular}

For QW width $\mathrm{L}=15 \mathrm{~nm}$, can be found following eigenvalues: $E_{1}=0.0459 \mathrm{eV}, E_{2}=0.156 \mathrm{eV}, E_{3}=0.295 \mathrm{eV}$, and for
$\mathrm{L}=6 \mathrm{~nm}$ can be found: $E_{1}=0.165 \mathrm{eV}, E_{2}=0.492 \mathrm{eV}, E_{3}=0.853 \mathrm{eV}$. Now it is possible the comparison of subband dispersion, obtained by the equation (8) and by the approximation (10). Graphical comparisons are shown in Figure 1a, b for the width of the QWs $6 \mathrm{~nm}$ and $15 \mathrm{~nm}$. The solid lines are derived from (8) and dashed are from the approximation (10). The graphic shows that the approximation (10) is quite exact in a wide range of values of $L$.
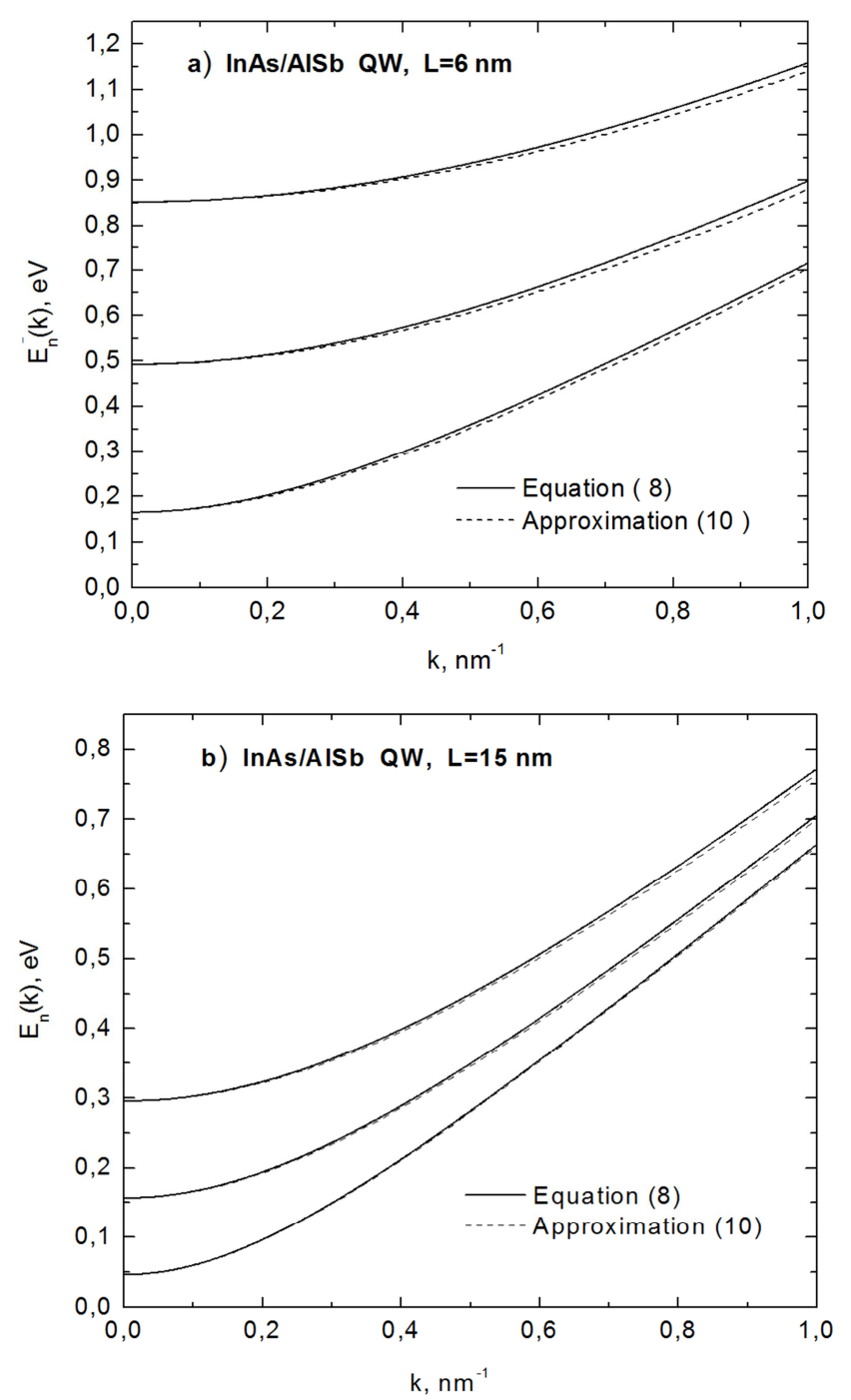

Figure 1. The dispersion of the first three subband for InAs/AlSb QW: a) $L=6 \mathrm{~nm} \mathrm{b)} L=15 \mathrm{~nm}$.

Now, solving the system of equations (12) and (13) can be constructed a dependence of DOS from the total concentration: $\rho_{s}\left(n_{s}\right)$. This dependence is shown in Figure 2 . In this figure of a dashed line it also shown the dependence $\rho_{s}\left(n_{s}\right)$, obtained in a parabolic approximation $E_{g A} \rightarrow \infty$. DOS values determined from experiment (see Table 1) are represented by triangular symbols.

It is can see from Figure 2, on the DOS calculation taking into account of nonparabolicity is important. With increasing concentration within each subband the DOS varies linearly, it increases also the value of the jump. DOS jump occurs at critical concentrations $n_{c 2}, n_{c 3}$, when the Fermi level crosses 
the bottom of the next subbands. Their value can be estimated from (13) assuming $E_{F}=E_{i}$. For example, when $\mathrm{L}=15 \mathrm{~nm}$, it is: $\quad n_{c 2}\left(E_{F}=E_{2}\right) \approx 1.55 \cdot 10^{12} \mathrm{~cm}^{-2}$, $n_{c 3}\left(E_{F}=E_{3}\right) \approx 7 \cdot 10^{12} \mathrm{~cm}^{-2}$. According to the experiment [5] filling the second subbands it begins at the critical concentration $n_{c 2}\left(E_{F}=E_{2}\right)=1.2 \times 10^{12} \mathrm{~cm}^{-2}$. Since in nonparabolic band the effective mass (and therefore DOS) is more than the parabolic case, the values of the critical concentrations $n_{c 2}, n_{c 3} \ldots$ also turns out more.

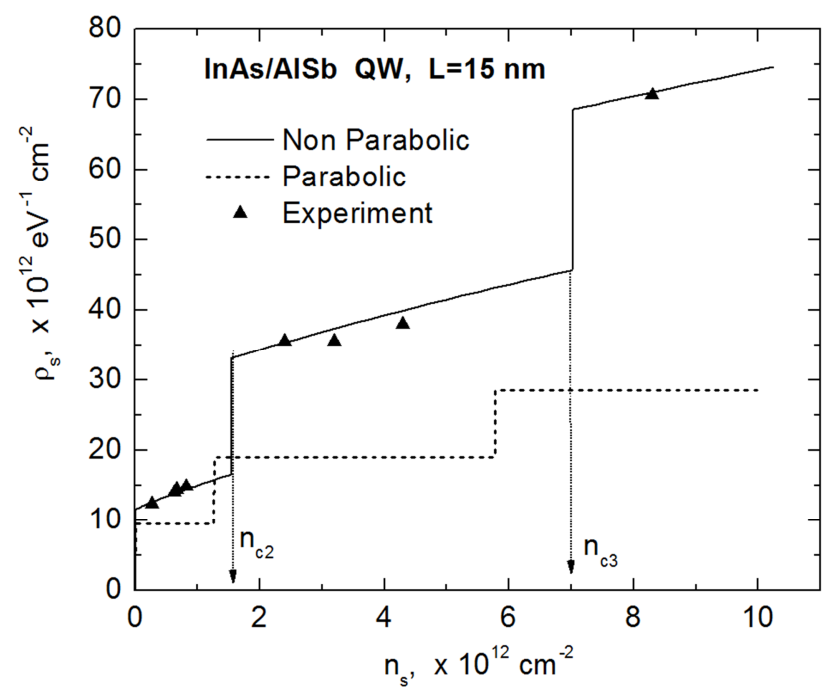

Figure 2. DOS dependence of electron gas from the total $2 D$ concentration found from system of equations (12) and (13) for InAs/AlSb QW by comparing the experimental data (see Table 1).

\section{Conclusion}

It is necessary will note, that the formula (5) is correct under condition, when $m_{c}\left(E_{F}\right)$ independent on the number of subbands $i$, strictly speaking. This condition is well carried out for InAs/AlSb QW [5, 11].

In this paper, the DOS of $2 \mathrm{D}$ electron gas was determined on the basis of experimental values of the effective mass at the Fermi level. It took the number of quantized levels lying below the Fermi level. Obtained on the basis of such data, the concentration dependence of the DOS is in good agreement with the model calculation of the DOS, as one can clearly see from Figure 2.

On the basis of a simple Kane model (7), a transcendental equation for subband dispersion (8) is given to a simpler form (10). Using this approximation, it was obtained simple relations (11), (12), (13) to calculate the concentration dependence of the DOS of 2D electron gas.

Regardless of proximity, obtained relations (11), (12), (13) are also useful in the study of the other equilibrium characteristics of the 2D electron gas in the QW hetero structure on the bases of semiconductor group $A_{3} B_{5}$.

\section{References}

[1] Shik, A. Y., Bakueva, L. G., Musikhin, S. F. and Rykov, S. A. (2001) Fizika nizkorazmernih system. SPb, Nauka, 160p. (in Russian).

[2] M. J. Yang, P. J. Lin-Chung, R. J. Wagner, J. R. Waterman, W. J. Moore, B. V. Shanabrook. Far-infrared spectroscopy in strained AlSb/InAs/AlSb quantum wells. Semicond. Sci. Technol. 8, S129 (1993) [DOI:10.1088/0268-1242/8/1S/029].

[3] C. Gauer, J. Scriba, A. Wixforth, J. P. Kotthaus, C. R. Bolognesi, C. Nguyen, B. Brar, H. Kroemer. Energydependent cyclotron mass in InAs/AlSb quantum wells. Semicond. Sci. Technol. 9, 1580 (1994) [DOI:10.1088/02681242/9/9/002].

[4] R. J. Warburton, C. Gauer, A. Wixforth, J. P. Kotthaus, B. Brar, H. Kroemer. Intersubband resonances in InAs/AlSb quantum wells: Selection rules, matrix elements, and the depolarization field. Phys. Rev. B53, 7903(1996) [DOI:10.1103/PhysRevB.53.7903].

[5] V. Ya. Aleshkin, V. I. Gavrilenko, A. V. Ikonnikov, Yu. G. Sadofyev, J. P. Bird, S. R. Jonhson, Y. -H. Zhang. A cyclotrone resonance in both doped and non-doped InAs/AlSb heterostructures with quantum wells. Fiz. Tekh. Poluprovodn. 39, 71-75(2005) (in Russian).

[6] I. V. Kukushkin, S. V. Meshkov, and V. B. Timofeev, "Twodimensional electron density of states in a transverse magnetic field," Soviet Physics Uspekhi, vol. 31, no. 6, pp. 511-534, 1988. (in Russian).

[7] V. Ya. Aleshkin, N. A. Bekin, M. N. Buyanova, B. N. Zvonkov, A. V. Murel. Investigation of the electron density of states in quantum wells and quantum dot arrays by $\mathrm{C}-\mathrm{V}$ method. Fiz. Tekh. Poluprovodn. 33, 1246-1252 (1999) (in Russian).

[8] N. V. Pavlov, G. G. Zegrya. Optical properties of hetero structures with deep quantum wells AlSb/InAs ${ }_{0.86} \mathrm{Sb}_{0.14} / \mathrm{AlSb}$. Fiz. Tekh. Poluprovodn. 48, 1217-1227 (2014) (in Russian).

[9] N. V. Pavlov, G. G. Zegrya. Influence of electrons and light holes energy spectrum nonparabolicity on optical properties of hetero structures with deep quantum wells $\mathrm{AlSb} / \mathrm{InAs}_{0.86} \mathrm{Sb}_{0.14} / \mathrm{AlSb}$. Fiz. Tekh. Poluprovodn. 49, 617627 (2015) (in Russian).

[10] Abdulazizov B. T., Baymatov P. J. The International Symposium "New Tendencies of Developing Fundamental and Applied Physics: Problems, Achievements, Prospectives". November 10-11, 2016, Tashkent, Uzbekistan. PP. 76-77. http://www.ips2016.uz.

[11] P. J. Baymatov, B. T. Abdulazizov. Concentration dependences of the electron effective mass, Fermi energy, and filling of subbands in doped InAs/AlSb quantum wells. Ukr. J. Phys. Vol. 62, No. 1, 46-50 (2017) [DOI:10.15407/ujpe62.01.0046]. 